\title{
Assessing Sensory Processing Disorders in a Child Guidance Clinic with Focus on ADHD
}

\author{
Dr. Piyali Ghosh ${ }^{1}$, Dr. Sayanti Ghosh ${ }^{1}$, Dr. Sonalika Mondal ${ }^{1}$, Mr. Subhendu Moulik ${ }^{2}$ \\ ${ }^{1}$ Department of Psychiatry. NRS Medical College. Kolkata \\ ${ }^{2}$ Therapy Unit, Department of Physical Medicine and Rehabilitation, NRS Medical College, Kolkata
}

\section{ABSTRACT}

Introduction : Sensory processing generally refers to the handling of sensory information by neural systems, including the functions of receptor organs and the peripheral and central nervous systems. Sensory processing difficulties occur in a host of neuro developmental problems like Autism Spectrum Disorder (ASD), and Attention Deficit Hyperactivity Disorder (ADHD) among several others. These can also occur in children considered otherwise normal. Aim : To assess prevalence and distribution of Sensory Processing Difficulties among children attending the Child Guidance Clinic with focus on ADHD (excluding ASD) in a tertiary care hospital in Kolkata. Methodology : All new cases attending the Child Guidance Clinic during study period were included. Serious comorbid physical illness requiring admission, refusal to give consent and cases diagnosed with Autism Spectrum Disorder, learning disorder, Intellectual Disabilities and Psychiatric sequelae of serious physical illness were exclusion factors. All children who were referred to the Child Guidance Clinic were routinely administered the Child Symptom Inventory (CSI), followed by detailed evaluation. Cases were selected as per inclusion and exclusion criteria. Final diagnosis regarding psychopathology was made on the basis of DSM-5 criteria. Following this, another researcher (Occupational therapist) who was blind to the diagnosis screened the patient for Sensory Processing Difficulties using the validated tools Winnie Dunn Short Sensory Profile (SSP). Results : Difference of mean SSP scores between two groups i.e. ADHD (mean score - 134.41) and others including ODD, CD, Mood disorders (mean score-153.10) was statistically significant. Conclusion : Clinicians should be aware that children with externalizing behaviors like ADHD may also have underlying sensory processing difficulties which may be masked. Hence evaluation of sensory processing difficulties should form an integral part of the assessment process in such conditions.

Key word : Sensory Processing Difficulties, ADHD, Occupational therapy.

ADHD is a frequent condition encountered among children and has been widely researched regarding its prevalence, proposed etiologies,

\section{Corrosponding Author}

Dr. Sayanti Ghosh, MD. Associate Professor

Department of Psychiatry. NRS Medical College. Kolkata

Email :dr_sayanti@rediffmail.com neurobiological explanations and comorbidities. Yet, there are several perplexing areas regarding their clinical manifestation which is an area of concern among researchers and clinicians. Sensory processing problems is an area which is commonly associated with Autism Spectrum Disorders, but it is not only confined to that area. Many children 
with ADHD also experience sensory overload which could explain certain symptoms of apparent inattention, hyperactivity and impulsivity and lead to melt-downs, inflexible thinking, impaired self-monitoring and emotional dyscontrol if not properly handled. Other than pharmacotherapy and behavioral management strategies, physicians and parents need to be aware of sensory issues which could cause such behavior. Occupational therapy has an important role to play alongside the conventional management techniques of ADHD but most importantly it must be tailor-made for every child having ADHD symptoms for their underlying yet undetected sensory problems.

Sensory processing generally refers to the handling of sensory information by neural systems, including the functions of receptor organs and the peripheral and central nervous systems. Problems with sensory processing have been noted in the literature since the 1960 's and $70^{\prime} \mathrm{s}^{(1)}$. The term sensory integration dysfunction was coined by the occupational therapist Jean Ayres to describe atypical social, emotional, motor and functional patterns of behavior related to poor processing of sensory stimuli ${ }^{(2)}$.

Sensory integration (SI) is the ability to organize sensory information to make an adaptive response $^{(3)}$. Some authors have suggested that SI should be referred to as multisensory integration ${ }^{(4)}$. Behaviors associated with sensory processing are not necessarily symptoms or abnormalities; these are differences and often abilities, such as enhanced perception. ${ }^{(5)}$ Hence apparently normal individuals may have such sensory processing differences too which may or may not affect their daily functioning skills. Sensory processing disorder (SPD) "affects the way the brain interprets the information that comes in and the response that follows, causing emotional, motor, and other reactions that are inappropriate and extreme" ${ }^{\prime \prime(6)}$

Parham and Mailloux ${ }^{(7)}$ outlined five functional impairments associated with SPD. These include decreased social participation and occupational engagement; decreased length, frequency, or complexity of adaptive responses (successful response to an environmental challenge); impaired self-confidence and/or self-esteem; poor daily life skills and reduced family life; and diminished fine-, gross-, and sensory-motor skill development. SPD can negatively affect development and functional abilities in behavior, emotional, motor, and cognitive domains ${ }^{(8)}$. Consequently, it is important to detect differences early with appropriate sensory processing assessment tools.

Sensory processing problem is of three types sensory modulation disorder (SMD), sensory based motor disorder and sensory discrimination disorder. SMD is of 3 types sensory hypersensitivity, sensory hyposensitivity and sensory seeking. In cases of Sensory over-responsivity or sensory hypersensitivity individuals respond to sensory stimuli in the way that is faster, longer, or more intense than what is expected ${ }^{(9)}$. This response can be towards any types of sensory stimuli. Sensory over-responsivity can be considered as an independent diagnosis ${ }^{(10)}$. For example, a child with tactile hypersensitivity or defensiveness might be defensive for hair-brushing and / or haircuts because she/he cannot tolerate it easily.

Another form of sensory processing problem is under-responsivity. Individuals with underresponsivity are unaware or they are slow to respond to sensory input. The third type is sensory seeking, where individuals crave or display excessive interest in sensory experiences. ${ }^{(11)}$ Sensory discrimination problems are another type of sensory processing problem characterized by difficulty interpreting the specific characteristics of sensory stimuli. ${ }^{(12)}$

The etiology of sensory processing is unknown. Genetic and familial factors have been suggested $^{(12)}$ Others suggest risk factors such as pre-, peri- and post-natal birth factors like maternal stress during pregnancy, jaundice, and allergies. ${ }^{(13)}$

Michael First ${ }^{(14)}$, editor of the DSM-IV TR, notes that three options for adding sensory processing 
disorders to the DSM-V have been discussed : 1) adding it as a new disorder; 2) adding it as a subtype that would apply to disorders such as Autistic Disorder or Attention-Deficit / Hyperactivity Disorder; or 3) adding a dimensional definition to the DSM-V appendix for "criteria sets and axes needing further study" in order to stimulate additional research. He further notes that the type of data that would be required include 1) evidence that sensory processing disorder describes a condition that is not adequately covered by an existing DSM-IV disorder; 2) evidence supporting its diagnostic validity; 3) evidence supporting its clinical utility; and 4) evidence supporting that there is a low risk of false positive diagnoses that might result if sensory processing disorder were to be added.

Sensory processing difficulties occur in a host of neuro-developmental problems like Autism Spectrum Disorder (ASD), Learning Disorders, Attention Deficit Hyperactivity Disorder (ADHD), Development Coordination disorders and such. These can also occur in children considered otherwise normal or neurotypical. Sensory processing problems seem to overlap numerous conditions, and there is uncertainty about whether it constitutes a distinct disorder or not. Estimated rates of sensory processing dysfunction for children with various disabilities have ranged as high as $40-88 \%{ }^{(15,16)}$. The prevalence and types of sensory processing impairments in children with ASD and ADHD are well documented in the literature. ${ }^{(17,18,19,20)}$

Compared with children without disabilities, children with ADHD exhibited greater difficulties in the sensorimotor domain, including visual and tactile processing ${ }^{(21,22)}$. From a sensory processing perspective, children with ADHD may not be receiving and processing sensory information properly and thereforemay have difficulty producing appropriate responses at both school and home and in the community. ${ }^{(17)} \mathrm{Neu}$ (1997) reported that more activity, less adaptability, and lower thresholds for sensory stimuli in infancy are related to a higher rate of diagnosis of ADHD in later stages ${ }^{(23)}$. Researchers have further identified vestibular sensory differences in children with attentional difficulties; moreover, these difficulties interfere with the children's performance in movement and skills development ${ }^{(2,24)}$. The literature supports the observations that children with ADHD have behavioural and conduct difficulties and disruptive behaviour disorders, particularly Oppositional Defiant disorder and Conduct disorder, as well as others. $^{(17,25,26)}$

It is clear that there is an alteration in the neural networks and a possible central role of dopamine for sensory problems that cannot be linked to specific cerebral lesions in children with ADHD. ${ }^{(27)}$ One of the prominent neuroanatomical markers for children and adolescents with ADHD is widespread cortical thickness reductions. ${ }^{(28)}$ Activities in resting state in sensory and sensory-related cortices in ADHD is significantly more than those of the control group ${ }^{(29)}$ Moreover, there is a connection between perception and action that is impaired in ADHD. ${ }^{(30)}$

Thus, it is seen that children with conduct disturbances come to the attention of service providers by virtue of their key handicapping condition, i.e. hyperactive problematic behaviours; however, when more closely examined, they often have other, co-morbid but undiagnosed disorders ${ }^{(31)}$. Knowledge of such co-morbidities may contribute not only to improved theoretical models of developmental disability, but also to the design of tailored inventions.

The identification of an association between sensory processing difficulties and behavioral problems of ADHD and other childhood disorders may have significant implications for clinical practice too. Currently, sensory processing is not routinely assessed in all children attending Child Guidance clinics. ${ }^{(32)}$ In cases clinically diagnosed as ASD sensory problems are expected and assessed prior to management strategies like Sensory Integration 
Therapy. However, the identification of sensory processing difficulties in clinically diagnosed ADHD cases may stand to strengthen the early management of ADHD children with behavior problems. This will help to promote optimal development, socially and academically.

\section{AIM:}

1. This study aims to assess prevalence and distribution of Sensory Processing Difficulties among children attending the Child Guidance Clinic (excluding ASD) in a tertiary care hospital in Kolkata with focus on ADHD.

2. To find out the association between perinatal factors and Sensory Processing Difficulties.

\section{MATERIALS AND METHODS}

Study design : Cross-sectional observational study

Study period: 6 months from date of commencement of the study

Study setting : Child Guidance Clinic (Department of Psychiatry) and Occupational therapy unit (Department of Physical Medicine, Regional Artificial Limb Fitting Centre), NRS Medical College, Kolkata

Selection criteria - All new cases attending the child Guidance Clinic during study period were included. Patients having serious comorbid physical illness requiring admission, refusal to give consent for this study and patients with Autism Spectrum Disorder, Intellectual Disabilities, Learning Disorder and Psychiatric sequelae of serious physical illness were excluded.

\section{STUDY TOOLS}

\section{SHORT SENSORY PROFILE BY WINNIE DUNN}

The primary variable in this study was reported behavioral sensory processing as measured by the $\mathrm{SSP}^{(33)}$. The SSP is a 38 -item caregiver report measure comprising the items that demonstrated the highest discriminative power of atypical sensory processing among all the items from the long version, the Sensory Profile ${ }^{(34)}$. The full SP, from which the norms were established, was standardized on 1,200 children. Items are scored on a 1-point to 5-point scale. One parent of each participant completed the $\mathrm{SSP}^{[35]}$, a reliable and valid parent-report measure of functional behaviors associated with abnormal responses to sensory stimuli. The seven SSP subtests are: (1) Tactile Sensitivity, (2) Movement Sensitivity, (3) Visual/Auditory Sensitivity, (4) Taste/Smell Sensitivity, (5) Auditory Filtering, (6) Low Energy/ Weak, and (7) Under-responsive/Seeks Sensation. The possible range of raw scores on the total scale is 38 to 190, with higher scores reflecting more normal performance. A score in the, typical performance! range indicates that the child does not have sensory processing difficulties, while scores in the "probable differencel or ", definite differencel ranges indicate that the child might have or does have sensory processing difficulties in that subscale.

The Total Score is the most sensitive indicator of sensory dysfunction. In this study, the SSP is most appropriate because in the early phase of its development the social-communication and motor items in the SP were eliminated. Thus, the SSP isolates sensory processing that is less confounded by items overlapping with the diagnostic features of autism. Initial studies of the validity of the SSP have demonstrated discriminate validity of $>95 \%$ in identifying children with and without sensory modulation difficulties ${ }^{(33)}$. Miller and colleagues also correlated dysfunctional sensory processing scores with abnormal psychophysiological responses to a series of sensory challenges ${ }^{(35)}$. Together, these findings provide initial support for use of the SSP as a valid measure of sensory processing.

\section{Child Symptom Inventory (CSI) - 4}

The CSI-4 is a behavior rating scale whose items correspond to the symptoms of disorders defined by the Diagnostic and Statistical Manual of Mental Disorders (4th ed. [DSM-IV]; American Psychiatric Association $^{(36)}$. 
Assessing Sensory Processing Disorders in ADHD

\section{The Vanderbilt ADHD Diagnostic Rating Scale (VADRS).}

The Vanderbilt ADHD Diagnostic Rating Scale (VADRS) is a psychological assessment tool for attention deficit hyperactivity disorder (ADHD) symptoms and their effects on behavior and academic performance in children ages 6-12. This measure was developed by Mark Wolraich at the Oklahoma Health Sciences Center and includes items related to Oppositional Defiant Disorder, Conduct Disorder, Anxiety, and Depression, disorders often comorbid with $\mathrm{ADHD}^{(37)}$.

\section{DSM- 5}

The Diagnostic and Statistical Manual of Mental Disorders, Fifth Edition (DSM-5) is the 2013 update to the Diagnostic and Statistical Manual of Mental Disorders, the taxonomic and diagnostic tool published by the American Psychiatric Association $(\mathrm{APA})^{(40)}$.

\section{SAMPLING METHOD}

Consecutive new patients have been taken attending the clinic within the study period and who met the inclusion criteria was taken.

\section{METHOD OF DATA COLLECTION}

Ethical clearance from the Institutional Ethics Committee has been obtained. Informed consent has been taken from the parents or guardian accompanying the child. After initial height, weight measurements and basic physical examination, all children were referred to the Child Guidance Clinic and all of them have been routinely administered the Child Symptom Inventory (CSI)-4, followed by detailed history, mental status examination and psychological assessment. 132 cases, which fulfilled the inclusion criteria, have been selected. Final diagnosis regarding psychopathology was made based on DSM-V criteria (10). For cases clinically diagnosed as having Externalizing disorders in the form of ADHD, ODD and Conduct disorders were also given the The Vanderbilt ADHD Diagnostic Rating Scale (VADRS). Following this, another researcher (Occupational therapist) who is blind to the DSM-V diagnosis screened the patients included in the study using the validated tool Winnie Dunn Short Sensory Profile.

ANALYSIS : Data was entered in MS Excel Sheet and subsequently analyzed using SPSS version 24 .

Descriptive analysis was computed in terms of mean and standard deviation with range for continuous variables and frequency with percentage for ordinal and nominal variables.

Association between variables was assessed by Independent Sample $\mathrm{t}$ test and Chi-square test.

Variables which did not fulfill the cut-off level ( $p>0.05$ ) were not considered to be reliable predictors of the dependent variable in the statistical model and were not entered for further analysis.

\section{RESULT}

We screened 249 new cases of age between 6 to 12 years attending the child guidance clinic of NRS medical college during the study period and 117 cases were excluded after taking detailed history, mental status examination and psychological assessment. All the cases were routinely administered CSI. 132 cases were selected who fulfilled exclusion criteria. Among them 93 cases were male child and 39 were female child. 42 cases had history of perinatal complications (eclampsia, seizure, any infections, prolonged labour, instrumental delivery, low birth weight, cord neck, birth asphyxia, early onset sepsis, poor Apgar Score). 80 cases were diagnosed as ADHDas per DSM- 5 criteria. 52 cases were diagnosed other disorder like communication disorder, motor disorder, separation anxiety disorder, disruptive mood dysregulation disorder etc. (Table 1) 
Table 1 : Socio-demagraphic Variables

\begin{tabular}{|c|c|c|c|c|c|}
\hline \multicolumn{2}{|c|}{ SEX } & \multicolumn{2}{|c|}{$\begin{array}{c}\text { PERINATAL } \\
\text { COMPLICATION }\end{array}$} & \multicolumn{2}{|c|}{$\begin{array}{l}\text { PSYCHIATRIC } \\
\text { DIAGNOSIS }\end{array}$} \\
\hline Male & FEMALE & PRESENT & ABSENT & ADHD & OTHERS \\
\hline 93 & 39 & 42 & 90 & 80 & 52 \\
\hline \multicolumn{6}{|c|}{ severity of sensory dysfunction } \\
\hline \multicolumn{2}{|c|}{ definite } & \multicolumn{3}{|c|}{ probable } & typical \\
\hline \multicolumn{2}{|c|}{52} & \multicolumn{3}{|c|}{43} & 37 \\
\hline
\end{tabular}

The mean SSP score was 141.77 with standard deviation of 18.486. The frequency distribution of the severity of sensory dysfunction showed (table no-1)
52 cases had definite problem of sensory dysfunction. 43 cases were within probable difference range and 37 cases were typical i.e. apparently normal sensory function.

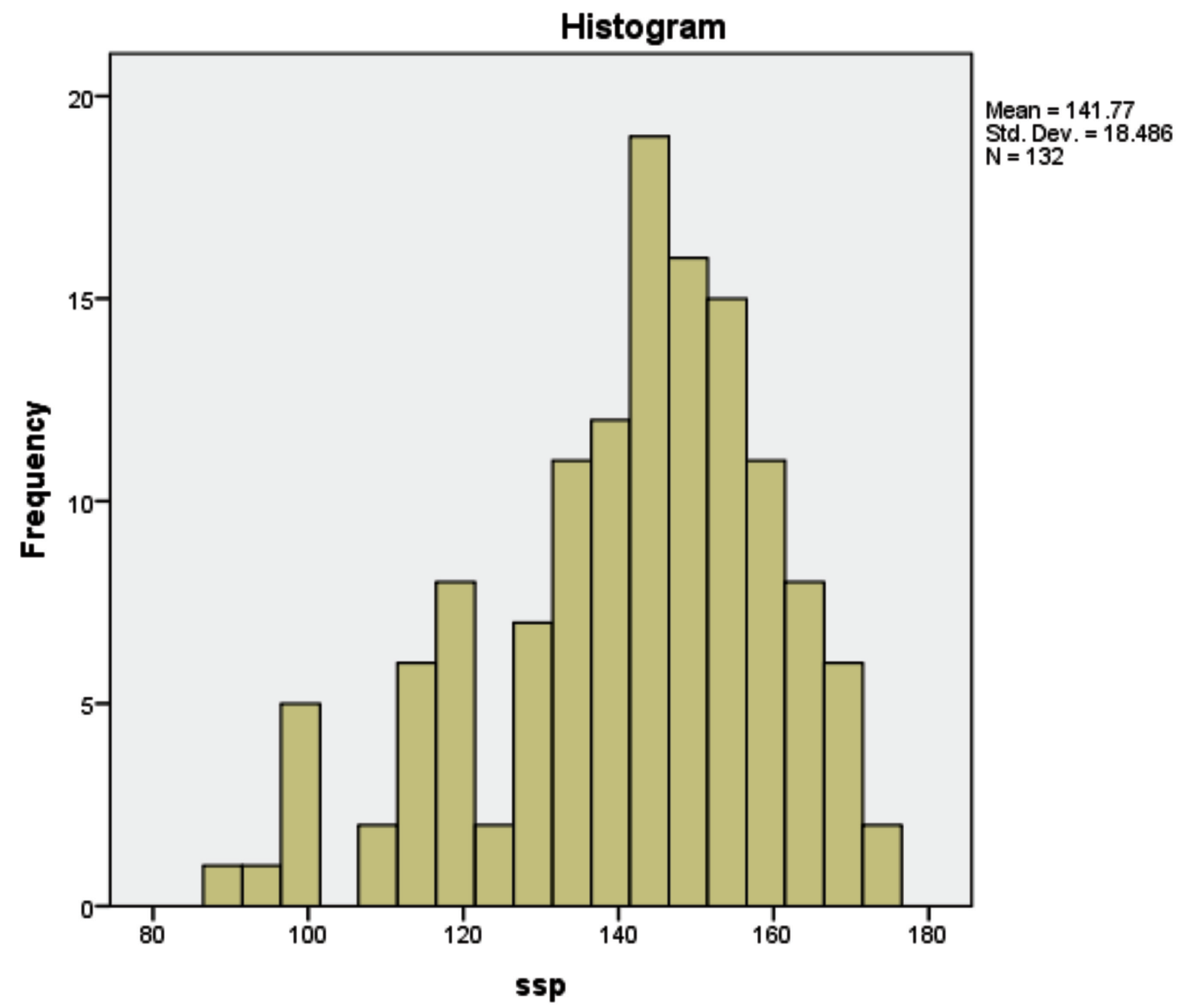

Figure 1 histogram of distribution of SSP score 
Assessing Sensory Processing Disorders in ADHD

The mean SSP score of the population diagnosed as ADHD was 134.41 i.e within definite problem of sensory dysfunction and mean score of other group was 153.10 (Table no 2)

\section{Table No - 2 : SSP Scores in Children}

\begin{tabular}{|c|c|c|c|c|c|}
\multicolumn{1}{c|}{} & Diag & $\mathrm{N}$ & Mean & Std. Deviation & Std. Error Mean \\
\hline \multirow{2}{*}{ SSP } & 1. ADHD & 80 & 134.41 & 17.731 & 1.982 \\
\cline { 2 - 6 } & 2. others & 52 & 153.10 & 13.231 & 1.835 \\
\hline
\end{tabular}

The result of independent sample Mann-Whitney $\mathrm{U}$ test showed a significant association ( $p$-value $<0.001$ ) between psychiatric diagnosis and mean SSP score.

Result of Chi-Square test shows a significant association (p-value 0.001) between perinatal complication and psychiatric diagnosis.

The result of independent sample Mann-Whitney U test shows no significant association ( $p$-value .461) between short sensory profile score across categories of perinatal complication.

\section{DISCUSSION :}

This study aimed to find the association between Sensory Processing Difficulties(SPD) among children attending the Child Guidance Clinic (excluding those diagnosed as Autism Spectrum Disorder, Intellectual Disabilities, Learning disabilities) with special focus on ADHD cases in a tertiary care hospital in Kolkata. 132 new cases were selected who fulfilled inclusion criteria. This was done with the goal of identifying whether and to what extent Sensory processing problems are present in children presenting to Child Clinic especially ADHD cases because it has been noted that besides ASD, sensory processing problems seem to be present in numerous other conditions, and there is uncertainty about whether it constitutes a distinct disorder or not. It has been studied that estimated rates of sensory processing dysfunction for children with various disabilities have ranged as high as $40-88 \%{ }^{(15,16)}$.

In this study selected cases were divided into two groups for analysis. First group was the children with diagnosis of ADHD and Second group included children with other diagnosis (ODD, CD, communication disorder, Childhood Depression, motor disorder, separation anxiety disorder, Disruptive Mood Dysregulation Disorder).

In the total sample of children in this study a high proportion of participants appeared to have sensory processing difficulties. $39.4 \%$ of them had 'definite' difference than 'typical' and $32.6 \%$ with probable difference. Previous studies on the prevalence of sensory processing difficulties in the community estimated a prevalence of $5.3-13.7 \%$, using a deliberately conservative cut-off ${ }^{(15)}$. Within the field of occupational therapy there is a prominent school of thought that suggests that some children can be clearly identified as having SPD either as part of or, in some cases independently of any other diagnoses ${ }^{(9)}$. There is evidence to show that certain children experience difficulties in the processing of sensory information compared to typical children ${ }^{(8,11,15,18,19,26)}$. Other than ASD, sensory processing problems seem to be present in numerous other conditions, and there is uncertainty about whether it constitutes a distinct disorder or not. Estimated rates of sensory processing dysfunction for children with various disabilities have ranged as high as $40-88 \%{ }^{(15,16)}$. So the high percentage of sensory processing difficulties in our study agrees with previous studies.

A possible explanation for a high prevalence of sensory processing difficulties in the total sample is the large number of cases with ADHD diagnosis. Similar findings were obtained in the study done by Cheung and $\mathrm{Siu}^{(39)}$ who specifically analyzed scores on each Sensory Profile item and found that the ADHD group scored lower than the control. 
In this study other externalizing behavioral disorders like ODD and CD were compiled in another group separately from ADHD cases but previous studies have found presence of sensory dysfunction within those behavioral childhood disorders too. Though results here suggest a significant difference of sensory processing difficulties in the two groups of study population aged 6-12 years sensory processing difficulties may coexist in both groups and possibly contribute to behavioral problems, although this would need to be examined longitudinally in order for any cause-effect relationships to be established. It may also be that a common neurological factor underlies the development of both externalizing behaviorandsensoryprocessingdifficulties. Behavior problems have been linked with dysfunction of the prefrontal cortex and the amygdala ${ }^{(40,41)}$, and EEGs in children with sensory processing difficulties suggest impaired sensory gating (the brain's ability to filter sensory information). However, the neural mechanisms underlying these phenomena remain unknown $^{(42)}$.

Though the present study has not emphasized on different areas of sensory dysfunction (tactile, taste and smell, movement, under responsive/seek sensation, auditory filtering, low energy/weak and visual/auditory sensitivity) it was found in previous studies that children with ADHD have more difficulties in tactile processing ${ }^{(21)}$. Tactile dysfunction comprises three types of tactile dysfunction including hypersensitivity to touch (tactile defensiveness), hyposensitivity to touch (under-responsive), and poor tactile perception and discrimination ${ }^{(43)}$. "Might not be aware that his/ her face or hands are dirty or even his/her runny nose" and "may not be aware that he/she has been touched, except that when he/she has been touched forcefully" two examples for hyposensitivity to touch. Two examples of tactile defensiveness are "doesn't like to brush his/her hair or is easily annoyed by it" and "react excessively to small cuts, or bites".

Certain behavior of hyperactive children like impulsivity, accident proneness could be explained on the basis of sensation seeking behavior.
The subtypes of ADHD are not different regarding auditory processing problems. However, the comorbidity of ODD in children with ADHD is a risk factor for auditory processing problems ${ }^{(44)}$.

In this study we found significant association between presence of perinatal complication and psychiatric diagnosis where the finding shows 34 cases with ADHD out of 80 cases had history of perinatal complication ( $42.5 \%$ of ADHD cases). This finding is consistent with the previous research review done by Marta Serati. et.al ${ }^{(45)}$ in which they found that preterm birth and low birth weight were the most important factor showing risk of developing ADHD in future.

However, this study could not find any significant association between perinatal complication and sensory processing difficulties though there are numerous studies which show significant association. This discrepancy in our study may be due to small sample size where we have very less no of cases with history of perinatal complications.

The significant association between sensory processing difficulties and attention deficit hyperactivity disorder has important implications for clinical practice. Clinicians should be aware that many children who present with symptoms of ADHD may have underlying sensory processing difficulties. Evaluation of sensory processing difficulties should form part of the assessment process, and consultation with an occupational therapist who has completed accredited training in sensory integration practice should be considered. Parents should also be informed about the future risk of developing ADHD in children with history of perinatal complication as well.

\section{Limitations}

- Sample size of this study was less.

- Most of the children scored in the high ranges because of thehigher number of study population in measures of externalizing behavior.

- The assessment of sensory processing could have been strengthened by the addition of the teacher-rated SSP. 
- Prevalence of sensory processing difficulties among apparently normal children could have been assessed as a comparator arm.

- Different areas of sensory processing difficulties have not been addressed in this study.

\section{CONCLUSION}

Very few Indian studies have explored the association between sensory processing difficulties and externalizing behaviors in children. It was hypothesized that the prevalence of sensory processing difficulties in this sample would be higher in ADHD cases and the results bore this out. Based on the SSP scores, the difference between two groups are significant. The findings suggest that further research into this association is warranted, as it may illuminate important management approaches for children with externalizing disorders especially ADHD.

\section{REFERENCES}

1. Bogdashina $\mathrm{O}$. What Exactly is Sensory Integration Dysfunction? Internet [On-line] 2003. Available : www. suite101.com/article.cfm/autism_world/97497.

2. Ayres J. Sensory Integration and the Child. Western Psychological Services; 1979.

3. Eeles A, Spittle AJ, Anderson PJ, Brown N, Lee K, Boyd R, et al. Assessment of sensory processing in infant: a systematic review. Dev Med Child Neurol (2013) 55 : 314-26.10.1111/ j.1469-8749.2012.04434.x[PubMed] [Cross Ref]

4. Crozier SC, Goodson JZ, Mackay ML, Synnes AR, Grunau RE, Miller SP, et al. Sensory processing patterns in children born very preterm. Am J Occup Ther (2015) 70(1):7001220050p17.10.5014/ajot.2016.018747 [PubMed] [Cross Ref]

5. Ayres J. Sensory Integration and Praxis Tests (SPIT). Los Angeles : Western Psychological Services; (1989).

6. Schaaf RC, Lane AE. Toward a best-practice protocol for assessment of sensory features in ASD. J Autism Dev Disord (2015) 45(5) : 1380-95.10.1007/s10803-014-2299-z [PubMed] [Cross Ref]

7. Brown T, Morrison IC, Stagnitti K. The convergent validity of two sensory processing scales used with school-age children : comparing the Sensory Profile and the Sensory Processing Measure. New Zeal J Occup Ther (2010) 57(2) : 56-65.

8. Baranek G, Little LM, Parham D, Ausderau KK, Sabatos-De Vito MG. Sensory features in autism spectrum disorders. 4th ed In : Volkmar FR, Rogers SJ, Paul R, Pelphrey KA, editors., editors. Handbook of Autism and Pervasive Developmental Disorders. New Jersey: John Wiley \& Sons, Inc; (2014). p. $378-408$.
9. Miller LJ, Anzalone ME, Lane SJ, Cermak SA, Osten ET. Concept evolution in sensory integration : a proposed nosology for diagnosis. Am J Occup Ther 2007; 61 : 135-140. PMID : 17436834.

10. Reynolds S, Lane SJ. Diagnostic validity of sensory overresponsivity : a review of the literature and case reports. J Autism Dev Disord 2008; 38 : 516-529. PMID : 17917804.

11. Ben-Sasson A, Cermak SA, Orsmond GI, Tager-Flusberg $\mathrm{H}$, Kadlec MB, Carter AS. Sensory clusters of toddlers with autism spectrum disorders : differences in affective symptoms. J Child Psychol Psychiatry 2008; 49 : 817-825. PMID : 18498344.

12. Miller LJ, Nielsen DM, Schoen SA, Brett-Green BA. Perspectives on sensory processing disorder: a call for translational research. Front Integr Neurosci 2009; 3 : 22PMID : 19826493.

13. May-Benson TA, Koomar JA, Teasdale A. Incidence of pre-, peri-, and post-natal birth and developmental problems of children with sensory processing disorder and children with autism spectrum disorder. Front Integr Neurosci 2009; 3 : 31PMID : 19936320

14. First M. Sensory Processing Disorder: Possible Pathways to DSM-V. Internet [On-line] 2005.

Available:www.spdnetwork.org/research/swg.first.html.

15. Ahn, R. R., Miller, L. J., Milberger, S., \& McIntosh, D. N. (2004). Prevalence of parents' perceptions of sensory processing disorders among kindergarten children. American Journal of Occupational Therapy, 58, 287-293

16. Talay-Ongan, A., \& Wood, K. (2000). Unusual sensory sensitivity in autism : A possible crossroad. International Journal of Disability, Development, and Education, 47, 201-212

17. Dunn, W., \& Bennett, D. (2002). Patterns of sensory processing in children with attention deficit hyperactivity disorder. Occupational Therapy Journal of Research, 22, 4-15.

18. Rogers, S. J., Hepburn, S., \& Wehner, E. (2003). Parent reports of sensory systems in toddlers with autism and those with other developmental disorders. Journal of Autism \& Developmental Disorders, 33, 631-642.

19. Tomchek, S. D., \& Dunn, W. (2007). Sensory processing in children with and without autism: A comparative study using the short sensory profile. American Journal of Occupational Therapy, 61, 190-200.

20. Yochman, A., Parush, S., \& Ornoy, A. (2004). Responses of preschool children with and without ADHD to sensory events in daily life. American Journal of Occupational Therapy, 58, 294-302.

21. Hern, K. L., \& Hynd, G. W. (1992). Clinical differentiation of the attention deficit disorder subtypes: Do sensorimotor deficits characterize children with ADD/WO? Archives of Clinical Neuropsychology, 7, 77-83.

22. Schaughency, E. A. (1986). Neuropsychological functioning of children diagnosed as attention deficit disorder with and without hyperactivity. (Doctoral dissertation, University of Georgia, 1986). Dissertation Abstracts International, 47, $2632 b$ 


\section{Assessing Sensory Processing Disorders in ADHD}

23. Neu, M. (1997). Irritable infants : Their childhood characteristics. Dissertation Abstracts International, 58(4B), 1805

24. Fisher, A. G., Murray, E. A., \& Bundy, A. C. (1991). Sensory integration theory and practice. Philadelphia : FA Davis.

25. Downey, K., Stelson, F., Pomerlearu, O., \& Giordani, B. (1997). Adult attention deficit hyperactivity disorder : Psychological test profiles in a clinical population. Journal of Nervous and Mental Disease, 185, 32-38.

26. Mangeot, S. D., Miller, L. J., McIntosh, D. N., McGrathClarke, J., Simon, J., Hagerman, R. J., et al. (2001). Sensory modulation dysfunction in children with attention-deficithyperactivity disorder. Developmental Medicine and Child Neurology, 43, 399-406.

27. Pasini A, D'agati E. Pathophysiology of NSS in ADHD. World J Biol Psychiatry 2009; 10 : 495-502. PMID : 19337883.

28. Narr KL, Woods RP, Lin J, Kim J, Phillips OR, Del'homme $\mathrm{M}$, et al. Widespread cortical thinning is a robust anatomical marker for attention-deficit/hyperactivity disorder. J Am Acad Child Adolesc Psychiatry 2009; 48 : 1014-1022. PMID : 19730275.

29. Tian L, Jiang T, Liang M, Zang Y, He Y, Sui M, et al. Enhanced resting-state brain activities in ADHD patients : a fMRI study. Brain Dev 2008; 30 : 342-348. PMID : 18060712.

30. Dockstader C, Gaetz W, Cheyne D, Tannock R. Abnormal neural reactivity to unpredictable sensory events in attentiondeficit/hyperactivity disorder. Biol Psychiatry 2009; 66 : 376-383. PMID : 19482264.

31. Cohen, N. J. 2001. Language Impairment and Psychopathology in Infants, Children, and Adolescents. Thousand Oaks, CA : Sage.

32. Gunn, T. E., B. D. Tavegia, B. M. Houskamp, L. B. McDonald, J. M. Bustrum, R. K. Welsh, and D. S. Mok. 2009. “Relationship between Sensory Deficits and Externalizing Behaviors in an Urban, Latino Preschool Population." Journal of Child and Family Studies 18 (6) : 653-661

33. McIntosh, D. N., L. J. Miller, V. Shyu, and W. Dunn. 1999. "Overview of the Short Sensory Profile (SSP)." In The Sensory Profile : Examiners Manual, edited by W. Dunn, 59-73. San Antonio, TX: The Psychological Corporation.

34. Dunn, W. 1999. Sensory Profile User's Manual. San Antonio, TX : Psychological Corporation

35. Miller, L. J., Reisman, J., McIntosh, D., \& Simon, J. (2001). An ecological model of sensory modulation. In S. Smith-Roley,
E. Blanche, \& R. C. Schaaf (Eds.), Understanding the nature of sensory integration with diverse populations (pp. 57-82). Los Angeles : Harcourt

36. Gadow, K. D., \& Sprafkin, J. Child Symptom Inventories manual. Stony Brook, NY : Checkmate Plus. 1994.

37. Collett, Brent R.; Ohan, Jeneva L.; Myers, Kathleen M. (September 2003). "Ten-Year Review of Rating Scales. $\mathrm{V}$ : Scales Assessing Attention-Deficit/Hyperactivity Disorder". Journal of the American Academy of Child \& Adolescent Psychiatry. 42 (9) : 1015-1037. doi : 10.1097/01. CHI.0000070245.24125.B6.

38. DSM-5. 5th ed, American Psychiatric Association,2013, DSM-V, doi-org.db29.linccweb.org/10.1176/appi.books, 9780890425596.dsm02.

39. Cheung PPP, Siu AMH. A comparison of patterns of sensory processing in children with and without developmental disabilities. Res Dev Disabil. 2009; 30(6) : 1468-1480. doi : 10.1016/j.ridd.2009.07.009. [PubMed] [CrossRef] [Google Scholar

40. Passamonti, L., G. Fairchild, I. M. Goodyer, G. Hurford, C. C. Hagan, J. B. Rowe, and A. J. Calder. 2010. "Neural Abnormalities in Early-Onset and Adolescence-Onset Conduct Disorder." Archives of general psychiatry 67 (7) : 729-738.

41. Ghanizadeh A. Screening signs of auditory processing problem: does it distinguish attention deficit hyperactivity disorder subtypes in a clinical sample of children? Int J Pediatr Otorhinolaryngol. 2009; 73 : 81-87. [PubMed]

42. Dawson, G. \& Watling, R. (2000) Interventions to facilitate auditory, visual and motor integration. A review of the evidence. Journal of Autism and Developmental Disorders, $30,415-421$

43. Ghanizadeh, A. 2008. "Tactile Sensory Dysfunction in Children with ADHD." Behavioural Neurology 20 (3-4): 107-112.

44. Reynolds S, Lane SJ. Sensory overresponsivity and anxiety in children with ADHD. Am J Occup Ther. 2009; 63 : 433-440. [PubMed]

45. Marta Serati, 1 Jennifer L. Barkin, 2 Giulia Orsenigo, 1 Alfredo Carlo Altamura, 1 and Massimiliano Buoli1, Research Review : The role of obstetric and neonatal complications in childhood attention deficit and hyperactivity disorder - a systematic review Journal of Child Psychology and Psychiatry, 2017. doi : 10.1111/jcpp.12779 